\title{
Assessment of Nutritional Status of Pregnant Women Attending Antenatal Care Cen- ters in Odo-Otin Local Government Area of Osun State, Nigeria
}

\section{Adedayo Adeogun ${ }^{{ }^{*}}$ and Caroline Adeoti ${ }^{2}$}

\author{
${ }^{1}$ Department of Food Science and Technology, Osun State Polytechnic, Iree, Nigeria \\ 2 Department of Ophthalmology, Ladoke Akintola University of Technology Teaching \\ Hospital , Osogbo, Nigeria
}

${ }^{*}$ Correspondence should be addressed to Adedayo Adeogun: adeogundayo@yahoo.com

Received o6 June 2019; Revised 19 July 2019; Accepted 23 July 2019

(C) 2019 Adeogun \& adeoti. Licensee Pan African Journal of Life Sciences. This is an Open Access article distributed under the terms of the Creative commons Attribution License (https://creativecommons.org/licenses/BY/4.0), which permits unrestricted use, distribution, and reproduction in any medium, provided the original work is properly cited.

Background: Good nutrition is a prerequisite for the national development of countries and for the well-being of every individual. Although problems related to poor nutrition affect the entire population, women and children are especially vulnerable because of their physiological and socio-economic characteristic. Pregnancy places extra nutritional demands on women and this may affect the development of the foetus and its health later in life. The nutritional demands however does not necessarily translate to increase in the amount of food to be consumed but certain nutrients such as protein, iron, folate, zinc and iodine are required in higher proportions to prevent anaemia and abortion. The general objective of this study was to assess the nutritional status of pregnant women aged 15-49 years that were attending antenatal care (ANC) at Primary Healthcare Centres, Odo-Otin Local Government Area, Osun State, Nigeria.

Methods: This was a cross-sectional descriptive study of 400 pregnant women attending ANC at Primary Health Care Centres in Odo-Otin Local Government Area of Osun State over a period of four weeks. Their anthropometric and bio-chemical data were determined. Their bio-data and dietary investigation were obtained using a questionnaire. Analysis was done using Statistical Package for Social Sciences (SPSS) version 20 and P was drawn at 0.05.

Results: One hundred and thirty two (33.3\%) of the participants were malnourished with their mid-upper arm circumference (MUAC) less than $23 \mathrm{~cm}$. Anaemia was found in 127(31.8\%) of participants and of this proportion, civil servants were more commonly affected 54 (42.5\%). Of the 90 participants that were civil servants, $46.5 \%$ were under -weight and they were the most malnourished while farmers had the least proportion (15.8\%) of underweight participants $(\mathrm{P}=0.001)$. More than half $(51.8 \%)$ of those who earned less than N10,000.00 were under-weight compared with $8.3 \%$ of those who earned more than N50,000.00 ( $\mathrm{P}=0.001)$. One hundred and eight (94.7\%) of those who skipped meals were under-weight compared to $6.3 \%$ of those who do not $(\mathrm{P}=0.001)$. Hence, $82.7 \%$ of those who skipped meals were anaemic as compared to17.3\% of those who do not $(\mathrm{P}=0.001)$.

Conclusion: The prevalence of malnutrition among pregnant women in Odo-Otin Local Government Area of Osun State, Nigeria was moderately low. However, it is worth noting that pregnant women who were Civil Servants were more malnourished .

Keywords: Nutritional status, pregnant women, antenatal care, Local Government Area 


\section{1.o INTRODUCTION}

The development of any country depend on the availability of good food and nutrients among other things for the well-being of every individual. The Council on Food and Nutrition of the American Medical Association defines nutrition as the science of food, the nutrients and the substance therein, their action, interaction and balance in reaction to health and disease: and the process by which the organization (i.e. body) ingests, digests, absorbs, transports and utilizes food substances [1]. Nutrition could also be described as the study of food values and its effect on the body as food is essential for optimum healthy living [2].

Pregnancy places extra nutritional demands on women which may affect the development of the foetus and its health later in life [3]. The nutritional demands however does not necessarily translate to increase in the amount of food to be consumed but certain nutrients such as protein, iron, folate, zinc and iodine are required in higher proportions to prevent anaemia. Anaemia during pregnancy is associated with higher rates of pre-natal morbidity, higher risk for miscarriage, prematurity, low birth weight, infants with low iron stores and babies higher risk of developing anaemia in the first month of life [3]. Studies have shown that most of the women in of sub-Sahara Africa, including Kenya, get pregnant under poor nutritional status which adversely affect their health and that of foetus [4].

One of the Suistainable Development Goals (SDGs) is to eradicate extreme poverty and hunger and to reduce child mortality and improve maternal health. It is less than a decade into SDGs and the maternal nutritional indices are not encouraging. Maternal prenatal morbidity and mortality has remained unacceptably high with not much impact by the government or the non-government agencies in Nigeria [5]. Maternal Mortality Rate (MMR) is unacceptability high in sub-Sahara Africa [6]. In Nigeria for example, MMR have been reported to be 630 deaths per 100,000 live births, thus ranking Nigeria 11th country in the world with highest MMR, among 184 countries [7].

A balanced, nourishing diet throughout pregnancy provides the nutrients needed to support fetal growth and development without depriving the mother the nutrients needed to maintain her own health. It also minimizes the risks of excess energy loss [7]. Usually, a woman whose diet is adequate before pregnancy is able to bear a full term viable infant [8]. Multiple micronutrient supplements taken with iron and folic acid can improve birth outcomes for women in low income countries [9]. These supplements reduce numbers of low birth weight of babies, premature and stillbirths in women [9]. Undernourished women can benefit from dietary education sessions, balanced energy and protein supplements [11]. This study therefore assess the nutritional status of pregnant women attending antenatal care centers in Odo-Otin Local Government Area of Osun State in order to make recommendations that will lead to improvement in nutritional habits of pregnant women and thereby prevent maternal and child mortality

\section{2.o METHODOLOGY}

The study was conducted in Odo-Otin Local Government area of Osun State, Nigeria. Osun State is one of the 36 States of the Federal Republic of Nigeria. It is an inland State in South-Western Nigeria with its capital in Osogbo $[11,12]$. A cross-sectional descriptive study was conducted to assess the anthropometric and bio-chemical measures of pregnant women aged 15-49 years attending antenatal clinics at the Local Government Area. The sample size was calculated using the prevalence of malnutrition in pregnant mothers in Nigeria found to be about 36\% [14]. The Cochran formula [15] for calculating a representative sample for proportions was used as follows:

$\mathrm{n}=\mathrm{Z} 2 \mathrm{pq} / \mathrm{e} 2$, Where; $\mathrm{n}$ is the desired sample size; $\mathrm{Z}$ is the standard normal deviate at 95\% confidence level (1.96); $p$ is the proportion of the target population estimated to have characteristic being measured $=36 \%$; $q$ is $1-p$; and $\mathrm{e}$ is the level of statistical significance set $=0.05$. Therefore; sample size of pregnant women $\mathrm{n}=1.962 \times 0.36$ (10.36)/0.052. Thus $n=354$. Due to the possibility of non - response, $10 \%$ was added to make a sample of 389 . A sample of 400 pregnant women was therefore used. Included in the study were pregnant women aged 15-49 years willing to participate in the study and who had been residents of Osun State for not less than six months prior to the study. Excluded pregnant women in the study included those less than 15 years and above 49 years, those not willing to participate, those with chronic diseases such as hypertension, diabetes, tuberculosis and HIV/ AIDS and those enrolled in intervention feeding or general food distribution. These intervention would have impact on nutritional status and dietary diversity and thus bias the result of the study.

The multi-stage sampling technique was used to select the participants as follows: Stage One: 8 out of the 15 wards in Odo-otin Local Government Area was randomly selected using simple random sampling technique (lottery method). Stage Two: Simple random sampling technique (lottery method) was used to select two Primary Health Care Centers from each of the 8 wards selected in stage 1 , making a total of 16 Primary Health Care Centers. Stage three: 25 pregnant women who were within 
the inclusion criteria were then selected from each Ward, using a simple random sampling technique (lottery method). This number was obtained by dividing the sample size by total number of Primary Health Care Centers that were randomly selected $(400 \div 16=25)$. The dependent variables measured were the Mid Upper Arm Circumference (MUAC), Body Mass Index and Haemoglobin level. These were assessed in second or third trimester at the same time of administering the questionnaire.

The tape measurement was used to measure the MUAC of the pregnant women, this was done to determine the nutritional status during the pregnancy. Measurement of MUAC was made while the woman stands up relaxed with her left arm hanging down with no clothing on the arm and this was done twice for each respondent to ensure accuracy. The left arm was used as it shows malnutrition while the right arm which is frequently used show lean muscle mass as a result of work. The MUAC was measured by first finding the mid-point of upper arm and then measuring the circumference. MUAC of the left arm was taken to nearest $0.1 \mathrm{~cm}[16]$.

The adult weighing scale (Bathroom scale) was used to record the weight. The participants were asked to step on scale with light dress, and foot wear removed. The reading on the scale was noted and recorded on the questionnaire. It was ensured that the scale was adjusted to the zero level to avoid error, and the weight was recorded to the nearest $0.1 \mathrm{Kg}$. The stadio-meter was used to measure the height of the pregnant woman. The respondent was asked to stand straight and tall with her head, shoulders and heels touching the wall. The number just below the measuring device was recorded to the nearest $0.1 \mathrm{~m}$.

The haemoglobin concentration was determined using the haemoglobin paper scale. One of the research assistants took the finger pricked blood samples of the respondents through using a lancet. The blood sample was dropped on the haemoglobin paper and allowed to dry. The blood spot was immediately matched against the given color standards and the corresponding value was recorded on the questionnaire. The Socio-demographic and economic variables determined includes age group, level of education, husband's educational status, number of household members, occupation, level of income per month, husband's occupation and number of wives. Dietary history was determined using the last 24 hours dietary recall. The participants were asked the type of food they have been eating for the past 24 hours.

Data was entered and analyzed using Statistical Package for Social Sciences (SPSS) version 20 and cleaned for abnormal values at regular intervals. A backup was done on an external hard drive. Univariate analysis was done by tabulating and reporting frequencies with their respective percentages. Bivariate analysis was done using the chisquare test with p-values less than 0.05 considered statistically significant for qualitative variables, while multivariate analysis was analyzed using binary logistic regression to control potential confounders.

\section{3.o RESULTS}

A total of 400 pregnant women participated in this study (Table 1). Majority of the participants were within age group 25 to 34 years $52.0 \%$, while $19.3 \%$ were within 15 to 24 years. The mean age of the participants was $30.88 \pm$ 7.14 years. Most (94.5\%) of them were married and had secondary school education (50.2\%), with $6.0 \%$ having no formal education. More than half of the participants' husbands (50.5\%) had secondary school education with $5.8 \%$ having no formal education. Majority (88.5\%) were Yoruba and were mostly Muslims (49.7\%) by religion and by occupation were mostly artisans (56.3\%). Almost half of the participants' husbands were artisans (48.5\%), while less than one fifth were civil servants (16.8\%). More than one third of the participants (36.0\%) earned less than N10,000 per month while majority $(55.5 \%)$ earned an average monthly income of N10,000 to N50,000. The mean average monthly income was N20,727.50 \pm 16773.24. More than three quarter $(77.5 \%)$ were in monogamous family setting while $90(22.5 \%)$ were in polygamous setting. All of the participants had height greater than $1.45 \mathrm{~m} 400$ (100\%), the mean height was $1.70 \pm 0.05 \mathrm{~m}$ and majority of them had weight greater than $50 \mathrm{~kg}$ were 368 (92\%) with mean weight of $61.30 \pm$ $7.83 \mathrm{~kg}$. Less than one third 114 (28.5\%) were underweight with the mean BMI of $20.99 \pm 2.35 \mathrm{~kg} / \mathrm{m} 2$. The breakdown of the demographic characteristics and socio-economic factors of the study participants are shown in table 1.

Table 2 shows the twenty four hour recall of dietary practice of the participating pregnant women in the study. More than one third of the participants (36.3\%) skipped meal. Of different reasons given for skipping meals, unavailability of food (44.8\%) was the most common. The type of food consumed by majority of the participants were starchy staples (17.4\%), consisting of cereals, white root and tuber while the least consumed was eggs 70 (3.8\%). The frequency of malnutrition and anemia in the study population is presented in table 3 . One third of the pregnant women 132 (33.3\%) were malnourished (MUAC less than $23 \mathrm{~cm}$ ), while 268 (67.0\%) were normal (MUAC greater than or equal to $23 \mathrm{~cm}$, with the mean of in the study population $23.77 \pm 3.29 \mathrm{~cm}$ ). Also, less than 
Table 1. Socio-demographicactors,economic characteristics of respondents

\begin{tabular}{|c|c|c|c|}
\hline Variable & Level & $\begin{array}{l}\text { Frequency } \\
(\mathrm{N}=400)\end{array}$ & $\begin{array}{l}\text { Percent- } \\
\text { age (\%) }\end{array}$ \\
\hline \multirow{4}{*}{$\begin{array}{l}\text { Age } \\
\text { group } \\
\text { (years) } \\
\text { Height }\end{array}$} & $15^{-24}$ & 77 & 19.3 \\
\hline & $25-34$ & 208 & 52.0 \\
\hline & $35-44$ & 115 & 28.7 \\
\hline & $\begin{array}{l}\text { Less than or equal } \\
\text { to } 1.45 \mathrm{~m}\end{array}$ & $\mathrm{O}$ & $\mathrm{O}$ \\
\hline \multirow{3}{*}{ Weight } & $\begin{array}{l}\text { Greater than } \\
1.45 \mathrm{~m}\end{array}$ & 400 & 100 \\
\hline & $\begin{array}{l}\text { Less than or equal } \\
\text { to } 5 \text { okg }\end{array}$ & 32 & 8 \\
\hline & Greater than $50 \mathrm{~kg}$ & 368 & 92 \\
\hline \multirow{2}{*}{ BMI } & Under weight & 114 & 28.5 \\
\hline & Normal & 286 & 71.5 \\
\hline \multirow{4}{*}{$\begin{array}{l}\text { Marital } \\
\text { status }\end{array}$} & Single & 22 & $5 \cdot 5$ \\
\hline & Married & 378 & 94.5 \\
\hline & None & 24 & 6.0 \\
\hline & Primary & 137 & $34 \cdot 3$ \\
\hline \multirow{2}{*}{$\begin{array}{l}\text { Level of } \\
\text { education }\end{array}$} & Secondary & 201 & 50.2 \\
\hline & Tertiary & 38 & 9.5 \\
\hline \multirow{2}{*}{ Religion } & Christianity & 184 & 46.0 \\
\hline & Islam & 199 & 49.7 \\
\hline \multirow{5}{*}{ Ethnicity } & Traditional & 17 & 4.3 \\
\hline & Yoruba & 354 & 88.5 \\
\hline & Igbo & 29 & $7 \cdot 3$ \\
\hline & Hausa & 17 & 4.2 \\
\hline & None & 23 & 5.8 \\
\hline \multirow{3}{*}{$\begin{array}{l}\text { Hus- } \\
\text { band's } \\
\text { educa- } \\
\text { tional } \\
\text { status }\end{array}$} & Primary & 86 & 21.5 \\
\hline & Secondary & 202 & 50.5 \\
\hline & Tertiary & 89 & 22.2 \\
\hline \multirow{5}{*}{$\begin{array}{l}\text { Occupa- } \\
\text { tion }\end{array}$} & Civil servant & 90 & 22.5 \\
\hline & Artisan & 225 & 56.3 \\
\hline & Farming & 53 & 13.2 \\
\hline & Unemployed & 29 & 7.2 \\
\hline & Civil servant & 67 & 16.8 \\
\hline \multirow{2}{*}{$\begin{array}{l}\text { Occupa- } \\
\text { tion of } \\
\text { the hus- } \\
\text { band }\end{array}$} & Artisan & 194 & 48.5 \\
\hline & Farming & 139 & $34 \cdot 7$ \\
\hline \multirow{3}{*}{$\begin{array}{l}\text { Average } \\
\text { monthly } \\
\text { income }\end{array}$} & $\begin{array}{l}\text { Less than } \\
\text { N10,000 }\end{array}$ & 144 & 36.0 \\
\hline & N10,000-50,000 & 222 & $55 \cdot 5$ \\
\hline & $\begin{array}{l}\text { More than } \\
\text { N50,000 }\end{array}$ & 34 & 8.5 \\
\hline $\begin{array}{l}\text { Family } \\
\text { type }\end{array}$ & $\begin{array}{l}\text { Monogamy } \\
\text { Polygamy }\end{array}$ & $\begin{array}{l}310 \\
90\end{array}$ & $\begin{array}{l}77.5 \\
22.5\end{array}$ \\
\hline
\end{tabular}

Note * represents multiple response question

one third of the pregnant women 127 (31.8\%) were anaemic (62 (48.8\%) had mild anaemia and 15 (11.8\%) were severely anaemic). The mean haemoglobin level was $10.41 \pm 2.13 \mathrm{~g} / \mathrm{dl}$.
Table 2. Twenty four hour recall of dietary practice in 400 respondents

\begin{tabular}{|c|c|c|c|}
\hline Variable & Level & Frequency & $\begin{array}{l}\text { Percentage } \\
(\%)\end{array}$ \\
\hline \multirow{2}{*}{$\begin{array}{l}\text { Do you skip } \\
\text { meal }\end{array}$} & Yes & 145 & 36.3 \\
\hline & No & 255 & 63.7 \\
\hline \multirow{2}{*}{$\begin{array}{l}\text { Reason for skip- } \\
\text { ping meal }\end{array}$} & $\begin{array}{l}\text { Unavailability } \\
\text { of food }\end{array}$ & 65 & 44.8 \\
\hline & Loss of appetite & 30 & 20.7 \\
\hline \multirow{11}{*}{$\begin{array}{l}\text { Food groups } \\
\text { consumed }\end{array}$} & Inconvenience & 50 & 34.5 \\
\hline & $\begin{array}{l}\text { Legumes, nuts } \\
\text { and seeds }\end{array}$ & 244 & 13.2 \\
\hline & $\begin{array}{l}\text { Starchy sta- } \\
\text { ples (cereals } \\
\text { and white root } \\
\text { and tuber) }\end{array}$ & 321 & 17.4 \\
\hline & $\begin{array}{l}\text { Dark green } \\
\text { leafy vegetables }\end{array}$ & 205 & 11.1 \\
\hline & Meat and fish & 203 & 11.0 \\
\hline & $\begin{array}{l}\text { Other vitamin A } \\
\text { rich fruits and } \\
\text { vegetables }\end{array}$ & 193 & 10.4 \\
\hline & $\begin{array}{l}\text { Other fruits and } \\
\text { vegetables }\end{array}$ & 125 & 6.8 \\
\hline & Organ meat & 108 & 5.8 \\
\hline & $\begin{array}{l}\text { Milk and milk } \\
\text { products }\end{array}$ & 80 & 4.3 \\
\hline & Eggs & 70 & 3.8 \\
\hline & Fat and oil & 300 & 16.2 \\
\hline
\end{tabular}

Table 3. Frequency of malnutrition and anemia

\begin{tabular}{|c|c|c|c|}
\hline & Level & $\begin{array}{l}\text { Frequency } \\
(\mathrm{N}=400)\end{array}$ & Percent (\%) \\
\hline $\begin{array}{l}\text { Mid upper } \\
\text { arm circum- } \\
\text { ference of } \\
\text { respondents }\end{array}$ & $\begin{array}{l}\text { Normal } \\
(\geq 23 \mathrm{~cm}) \\
\text { Malnour- } \\
\text { ished } \\
(<23 \mathrm{~cm})\end{array}$ & 132 & 67.0 \\
\hline \multirow{2}{*}{$\begin{array}{l}\text { Anaemic status } \\
\text { of the respond- } \\
\text { ents }\end{array}$} & $\begin{array}{l}\text { Not anae- } \\
\text { mic }\end{array}$ & 273 & 68.2 \\
\hline & Anaemic & 127 & 31.8 \\
\hline \multirow{3}{*}{$\begin{array}{l}\text { Severity of } \\
\text { anaemia } \\
\text { among re- } \\
\text { spondents }\end{array}$} & Mild & 62 & 48.8 \\
\hline & Moderate & 50 & 39.4 \\
\hline & Severe & 15 & 11.8 \\
\hline
\end{tabular}

The relationship between socio-demographic factors of respondents, and their BMI are shown in table 4 . Statistical significant relationship was not observed when BMI was compared to age, marital status, level of education, religion, ethnicity and husband's educational status. On the other hand occupation had significant 
effect on BMI with the proportion of underweight participants (46.5\%) been highest among civil servants and least $(15.8 \%)$ among farmers $\left(\mathrm{X}^{2}=119.930, \mathrm{P}=\right.$ o.001). Also participant's husband occupation contributed significantly to BMI with civil servants recording the highest (46.5\%) proportion of underweight pregnant women, while farmers had the least (21.1\%) $\left(\mathrm{X}^{2}=\right.$ 101.268, $\mathrm{P}=0.003$ ).

Similarly, average monthly income also had a significant effect on the participants BMI with those earning less than N10,000 per month being more underweight (51.8\%) compared with those earning more than N50,000 (9.6\%) $\left(\mathrm{X}^{2}=19.449, \mathrm{P}=0.001\right)$. Family type also produce a significant effect on BMI with pregnant women from polygamous family settings (66.7\%) being more underweight compared to those in monogamous settings (33.3\%) $\left(\mathrm{X}^{2}=13.036, \mathrm{P}=0.001\right)$. Pregnant women who skipped meals were more underweight (94.7\%) compared with those who did not $5.3 \%\left(\chi^{2}=66.247, p=0.001\right)$.

Table 5 shows the relationship between socio demographic factors, economic characteristics and MUAC of the respondents. A significant association $(p=0.0001)$ was observed when MUAC was compared to occupation with civil servants (47.7\%) being the most malnourished. Also occupation of husband, Average monthly income, family type and skipping of meals produced a significant association $(p=0.001)$ on MUAC with civil servant (37.2\%), average monthly income less than N10,000 (50.8\%), polygamy family type (60.6\%) and participants who skipped meal (79.5\%) respectively, being the most malnourished. On the other hand Age group, marital status, level of education, religion, ethnicity and husband's educational status did not produce any significant effect on MUAC.

The relationship between socio demographic factors, economic characteristics, and anaemic status is shown in table 6 . The highest proportion of participants with anaemia were found in the age group 25 to 34 years $(48.0 \%$, $\mathrm{p}=0.033$ ), civil servants $(42.5 \%, \mathrm{p}=0.0001)$, women whose husbands are artisan (37.8\%; $\mathrm{p}=0.000)$, participants whose earnings are less than N10,00o (48.0\%; $\mathrm{p}=$ o.0001), those with polygamy family type $(52.8 \%$; $\mathrm{p}=$ 0.001) and those who skipped meals (82.7\%; X2= 28.846, $\mathrm{P}=0.001$ ).
Table 4. Relationship between socio-demographic factors of respondents, and body mass index

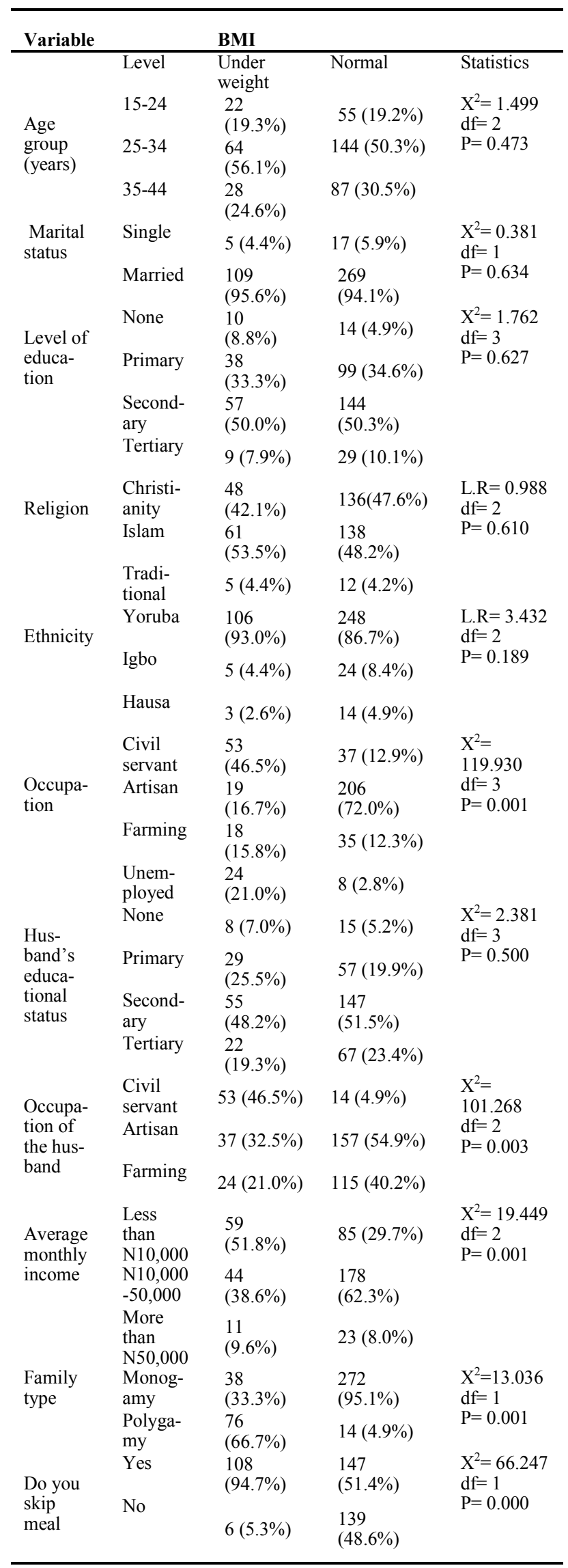


Table 5. Relationship between socio-demographic factors, economic characteristics and MUAC of the respondents.

\begin{tabular}{|c|c|c|c|c|}
\hline \multicolumn{5}{|c|}{ MUAC } \\
\hline Variable & Level & Normal & $\begin{array}{l}\text { Malnour- } \\
\text { ished }\end{array}$ & Statistics \\
\hline \multirow[t]{2}{*}{$\begin{array}{l}\text { Do you } \\
\text { skip meal }\end{array}$} & Yes & $\begin{array}{l}150 \\
(56.0 \%)\end{array}$ & $105(79.5 \%)$ & \multirow{5}{*}{$\begin{array}{l}\mathrm{X}^{2}= \\
21.270 \\
\mathrm{df}=1 \\
\mathrm{P}= \\
0.000 \\
\mathrm{X}^{2}= \\
4.917 \\
\mathrm{df}=2 \\
\mathrm{P}= \\
\text { o.o86 }\end{array}$} \\
\hline & No & $\begin{array}{l}118 \\
(44.0 \%)\end{array}$ & 27 (20.5\%) & \\
\hline \multirow{3}{*}{$\begin{array}{l}\text { Age } \\
\text { group } \\
\text { (years) }\end{array}$} & $15-24$ & $\begin{array}{l}55 \\
(20.5 \%)\end{array}$ & $22(16.7 \%)$ & \\
\hline & $25-34$ & $\begin{array}{l}129 \\
(48.2 \%)\end{array}$ & $79(59.8 \%)$ & \\
\hline & $35-44$ & $\begin{array}{l}84 \\
(31.3 \%)\end{array}$ & $31(23.5 \%)$ & \\
\hline \multirow[t]{2}{*}{$\begin{array}{l}\text { Marital } \\
\text { status }\end{array}$} & Single & $\begin{array}{l}16 \\
(6.0 \%)\end{array}$ & $6(4.5 \%)$ & \multirow{6}{*}{$\begin{array}{l}\mathrm{X}^{2}= \\
0.345 \\
\mathrm{df}=1 \\
\mathrm{P}=0.557 \\
\mathrm{X}^{2}= \\
4.996 \\
\mathrm{df}=3 \\
\mathrm{P}=0.172\end{array}$} \\
\hline & Married & $\begin{array}{l}252 \\
\text { (94.0\%) }\end{array}$ & $126(95.5 \%)$ & \\
\hline \multirow{4}{*}{$\begin{array}{l}\text { Level of } \\
\text { education }\end{array}$} & None & $12(4.5 \%)$ & $12(9.1 \%)$ & \\
\hline & Primary & $\begin{array}{l}98 \\
(36.5 \%)\end{array}$ & $39(29.6 \%)$ & \\
\hline & Secondary & $\begin{array}{l}131 \\
(48.9 \%)\end{array}$ & $70(53.0 \%)$ & \\
\hline & Tertiary & $\begin{array}{l}27 \\
(10.1 \%)\end{array}$ & $11(8.3 \%)$ & \\
\hline \multirow[t]{3}{*}{ Religion } & $\begin{array}{l}\text { Christiani- } \\
\text { ty }\end{array}$ & $\begin{array}{l}122 \\
(45.5 \%)\end{array}$ & $62(47.0 \%)$ & \multirow{3}{*}{$\begin{array}{l}\mathrm{X}^{2}= \\
\mathrm{O} .732 \\
\mathrm{df}=2 \\
\mathrm{P}= \\
0.693\end{array}$} \\
\hline & Islam & $\begin{array}{l}133 \\
(49.6)\end{array}$ & $66(50.0 \%)$ & \\
\hline & Traditional & $13(4.9 \%)$ & $4(3.0 \%)$ & \\
\hline \multirow[t]{3}{*}{ Ethnicity } & Yoruba & $\begin{array}{l}236 \\
(88.1 \%)\end{array}$ & $\begin{array}{l}118 \\
(89.4 \%)\end{array}$ & \multirow{3}{*}{$\begin{array}{l}\mathrm{X}^{2}= \\
0.443 \\
\mathrm{df}=2 \\
\mathrm{P}=0.801\end{array}$} \\
\hline & Igbo & $21(7.8 \%)$ & $8(6.1 \%)$ & \\
\hline & Hausa & $11(4.1 \%)$ & $6(4.5 \%)$ & \\
\hline \multirow{4}{*}{$\begin{array}{l}\text { Hus- } \\
\text { band's } \\
\text { educa- } \\
\text { tional } \\
\text { status }\end{array}$} & None & $\begin{array}{l}16 \\
(6.0 \%)\end{array}$ & $7(5 \cdot 3 \%)$ & \multirow{4}{*}{$\begin{array}{l}\mathrm{X}^{2}= \\
2.043 \\
\mathrm{df}=3 \\
\mathrm{P}=0.563\end{array}$} \\
\hline & Primary & $\begin{array}{l}53 \\
(19.8 \%)\end{array}$ & $33(25.0 \%)$ & \\
\hline & Secondary & $\begin{array}{l}141 \\
(52.6 \%)\end{array}$ & $61(46.2 \%)$ & \\
\hline & Tertiary & $\begin{array}{l}58 \\
(21.6 \%)\end{array}$ & $31(23.5 \%)$ & \\
\hline \multirow{4}{*}{$\begin{array}{l}\text { Occupa- } \\
\text { tion }\end{array}$} & $\begin{array}{l}\text { Civil serv- } \\
\text { ant }\end{array}$ & $\begin{array}{l}27 \\
(10.1 \%)\end{array}$ & $63(47.7 \%)$ & \multirow{4}{*}{$\begin{array}{l}\mathrm{X}^{2}= \\
138.200 \\
\mathrm{df}=3 \\
\mathrm{P}= \\
0.0001\end{array}$} \\
\hline & Artisan & $\begin{array}{l}200 \\
(74.6 \%)\end{array}$ & $25(18.9 \%)$ & \\
\hline & Farming & $\begin{array}{l}35 \\
(13.1 \%)\end{array}$ & $18(13.6 \%)$ & \\
\hline & $\begin{array}{l}\text { Unem- } \\
\text { ployed }\end{array}$ & $6(2.2 \%)$ & $26(19.8 \%)$ & \\
\hline \multirow{3}{*}{$\begin{array}{l}\text { Occupa- } \\
\text { tion of } \\
\text { the hus- } \\
\text { band }\end{array}$} & $\begin{array}{l}\text { Civil serv- } \\
\text { ant }\end{array}$ & $18(6.7 \%)$ & $49(37.2 \%)$ & \multirow{3}{*}{$\begin{array}{l}\mathrm{X}^{2}= \\
58.941 \\
\mathrm{df}=2 \\
\mathrm{P}= \\
0.0001\end{array}$} \\
\hline & Artisan & $\begin{array}{l}148 \\
(55.2 \%)\end{array}$ & $46(34.8 \%)$ & \\
\hline & Farming & $\begin{array}{l}102 \\
(38.1 \%)\end{array}$ & $37(28.0 \%)$ & \\
\hline \multirow{3}{*}{$\begin{array}{l}\text { Average } \\
\text { monthly } \\
\text { income }\end{array}$} & $\begin{array}{l}\text { Less than } \\
\text { N10,o0o }\end{array}$ & $\begin{array}{l}77 \\
(28.7 \%)\end{array}$ & $67(50.8 \%)$ & \multirow{3}{*}{$\begin{array}{l}\mathrm{X}^{2}= \\
19.482 \\
\mathrm{df}=2 \\
\mathrm{P}= \\
0.0001\end{array}$} \\
\hline & $\begin{array}{l}\text { N10,000- } \\
50,000\end{array}$ & $\begin{array}{l}168 \\
(62.7 \%)\end{array}$ & $54(40.9 \%)$ & \\
\hline & $\begin{array}{l}\text { More than } \\
\text { N50,000 }\end{array}$ & $\begin{array}{l}23 \\
(8.6 \%)\end{array}$ & $11(8.3 \%)$ & \\
\hline \multirow[t]{2}{*}{$\begin{array}{l}\text { Family } \\
\text { type }\end{array}$} & Monogamy & $\begin{array}{l}258 \\
(96.3 \%)\end{array}$ & $52(39.4 \%)$ & \multirow{2}{*}{$\begin{array}{l}X^{2}=164 . \\
O 60 \\
d f=1 \\
P= \\
0.0001\end{array}$} \\
\hline & Polygamy & $10(3.7 \%)$ & $80(60.6 \%)$ & \\
\hline
\end{tabular}

Table 6. Relationship between socio demographic factors, economic characteristics, and anemic status

\begin{tabular}{|c|c|c|c|c|}
\hline \multirow[b]{2}{*}{ Variable } & \multirow[b]{2}{*}{ Level } & \multicolumn{2}{|c|}{ ANAEMIC STATUS } & \multirow{5}{*}{$\begin{array}{l}\text { Statis- } \\
\text { tics } \\
\mathrm{X}^{2}= \\
6.850 \\
\mathrm{df}=2 \\
\mathrm{p}= \\
0.033\end{array}$} \\
\hline & & $\begin{array}{l}\text { Not anae- } \\
\text { mic }\end{array}$ & Anaemic & \\
\hline \multirow{3}{*}{$\begin{array}{l}\text { Age } \\
\text { group } \\
\text { (years) }\end{array}$} & $15-24$ & $43(15.8)$ & $\begin{array}{l}34 \\
(26.8 \%)\end{array}$ & \\
\hline & $25-34$ & $147(53.8)$ & $61(48.0 \%)$ & \\
\hline & $35-44$ & $83(30.4)$ & $32(25.2 \%)$ & \\
\hline \multirow[t]{2}{*}{$\begin{array}{l}\text { Marital } \\
\text { status }\end{array}$} & Single & $15(5 \cdot 5 \%)$ & $7(5.5 \%)$ & $\begin{array}{l}\mathrm{X}^{2}= \\
0.001\end{array}$ \\
\hline & Married & $\begin{array}{l}258 \\
(94.5)\end{array}$ & $\begin{array}{l}120 \\
(94.5 \%)\end{array}$ & $\begin{array}{l}\mathrm{df}=1 \\
\mathrm{p}= \\
0.994\end{array}$ \\
\hline \multirow{4}{*}{$\begin{array}{l}\text { Level of } \\
\text { educa- } \\
\text { tion }\end{array}$} & None & $\begin{array}{l}17 \\
(6.2 \%)\end{array}$ & $7(5.5 \%)$ & $\begin{array}{l}\mathrm{X}^{2}= \\
1.331\end{array}$ \\
\hline & Primary & $96(35.2)$ & $\begin{array}{l}41 \\
(32.3 \%)\end{array}$ & $\begin{array}{l}\mathrm{df}=3 \\
\mathrm{P}=\end{array}$ \\
\hline & Secondary & $137(50.2)$ & $\begin{array}{l}64 \\
(50.4 \%)\end{array}$ & 0.722 \\
\hline & Tertiary & $23(8.4 \%)$ & $15(11.8 \%)$ & \\
\hline \multirow[t]{3}{*}{ Religion } & Christianity & $127(46.6)$ & $57(44.9 \%)$ & $\begin{array}{l}\mathrm{X}^{2}= \\
3.684\end{array}$ \\
\hline & Islam & $\begin{array}{l}138 \\
(50.5 \%\end{array}$ & $\begin{array}{l}61 \\
(48.0 \%)\end{array}$ & $\begin{array}{l}\mathrm{df}=2 \\
\mathrm{P}= \\
\end{array}$ \\
\hline & Traditional & $8(2.9 \%)$ & $9(7.1 \%)$ & \\
\hline \multirow[t]{3}{*}{ Ethnicity } & Yoruba & $\begin{array}{l}245 \\
(89.7)\end{array}$ & $\begin{array}{l}109 \\
(85.8 \%)\end{array}$ & $\begin{array}{l}\mathrm{X}^{2}= \\
2.482\end{array}$ \\
\hline & Igbo & $16(5 \cdot 9 \%)$ & $\begin{array}{l}13 \\
(10.3 \%)\end{array}$ & $\begin{array}{l}\mathrm{df}=2 \\
\mathrm{P}=\end{array}$ \\
\hline & Hausa & $12(4.4 \%)$ & $5(3.9 \%)$ & \\
\hline \multirow{5}{*}{$\begin{array}{l}\text { Hus- } \\
\text { band's } \\
\text { educa- } \\
\text { tional } \\
\text { status }\end{array}$} & None & $16(5.9 \%)$ & $7(5.5 \%)$ & $\begin{array}{l}\mathrm{X}^{2}= \\
2.752\end{array}$ \\
\hline & Primary & $63(23.1)$ & $23(18.1 \%)$ & $\begin{array}{l}\mathrm{df}=3 \\
\mathrm{P}=\end{array}$ \\
\hline & Secondary & $\begin{array}{l}139 \\
(50.9)\end{array}$ & $\begin{array}{l}63 \\
(49.6 \%)\end{array}$ & 0.431 \\
\hline & Tertiary & $55(20.1)$ & $\begin{array}{l}34 \\
(26.8 \%)\end{array}$ & \\
\hline & Civil servant & $36(13.2)$ & $\begin{array}{l}54 \\
(42.5 \%)\end{array}$ & $\begin{array}{l}X^{2}= \\
133.971\end{array}$ \\
\hline \multirow[t]{3}{*}{$\begin{array}{l}\text { Occupa- } \\
\text { tion }\end{array}$} & Artisan & $\begin{array}{l}202 \\
(74.0)\end{array}$ & $\begin{array}{l}23 \\
(18.2 \%)\end{array}$ & $\begin{array}{l}\mathrm{df}=3 \\
\mathrm{P}=\end{array}$ \\
\hline & Farming & $32(11.7)$ & $21(16.5 \%)$ & \\
\hline & Unemployed & 3 (1.1\%) & $\begin{array}{l}29 \\
(22.8 \%)\end{array}$ & \\
\hline \multirow{3}{*}{$\begin{array}{l}\text { Occupa- } \\
\text { tion of } \\
\text { the hus- } \\
\text { band }\end{array}$} & Civil servant & $\begin{array}{c}30 \\
(11.0 \%)\end{array}$ & 37 (29.1\%) & $\begin{array}{l}\mathrm{X}^{2}= \\
21.585\end{array}$ \\
\hline & Artisan & $\begin{array}{l}146 \\
(53.5 \%)\end{array}$ & $48(37.8 \%)$ & \\
\hline & Farming & $\begin{array}{c}97 \\
(35.5 \%)\end{array}$ & $42(33.1 \%)$ & 0.000 \\
\hline \multirow{3}{*}{$\begin{array}{l}\text { Average } \\
\text { monthly } \\
\text { income }\end{array}$} & $\begin{array}{l}\text { Less than } \\
\text { N10,000 }\end{array}$ & $83(30.4)$ & $\begin{array}{l}61 \\
(48.0 \%)\end{array}$ & $\begin{array}{l}\mathrm{X}^{2}= \\
28.085\end{array}$ \\
\hline & $\begin{array}{l}\text { N10,000- } \\
50,000\end{array}$ & $175(64.1)$ & $\begin{array}{l}47 \\
(37.0 \%)\end{array}$ & $\begin{array}{l}\mathrm{df}=2 \\
\mathrm{P}=\end{array}$ \\
\hline & $\begin{array}{l}\text { More than } \\
\text { N50,000 }\end{array}$ & $\begin{array}{c}15 \\
(5 \cdot 5 \%)\end{array}$ & $19(15.0 \%)$ & 0.0001 \\
\hline \multirow[t]{2}{*}{$\begin{array}{l}\text { Family } \\
\text { type }\end{array}$} & Monogamy & $\begin{array}{l}250 \\
(91.6)\end{array}$ & $\begin{array}{l}60 \\
(47.2 \%)\end{array}$ & $\begin{array}{l}\mathrm{X}^{2}=97 . \\
687\end{array}$ \\
\hline & Polygamy & $\begin{array}{c}23 \\
(8.4 \%)\end{array}$ & $\begin{array}{l}67 \\
(52.8 \%)\end{array}$ & $\begin{array}{l}\mathrm{df}=1 \\
\mathrm{P}= \\
0.001\end{array}$ \\
\hline \multirow{3}{*}{$\begin{array}{l}\text { Do you } \\
\text { skip meal }\end{array}$} & Yes & $\begin{array}{l}150 \\
(54.9)\end{array}$ & $\begin{array}{l}105 \\
(82.7 \%)\end{array}$ & $\begin{array}{l}\mathrm{X}^{2}= \\
28.846\end{array}$ \\
\hline & No & & & $\mathrm{df}=1$ \\
\hline & & $123(45 \cdot 1)$ & $\begin{array}{l}22 \\
(17 \cdot 3 \%)\end{array}$ & $\begin{array}{l}\mathrm{P}= \\
0.000\end{array}$ \\
\hline
\end{tabular}




\subsection{DISCUSSION}

This study shows that pregnant women who are civil servants in Osun State are more likely to be underweight and malnourished compared to those who are farmers as observed in their significantly different BMI. This is contrary to findings in other studies by NDHS [14], and Ikechukwu et al [17] that reported higher mean BMI among civil servants in their study which was attributed to the higher purchasing power that possibly led to excessive calorie intake in comparison to farmers and artisans. On the contrary, the findings of this study showed lower mean BMI in women who are civil servants in comparison to those who were farmers and artisans. This may be explained by the prevailing economic situation in Osun State in which civil servants have been previously on modulated salaries for more than a year.

The prevalence of malnutrition observed in this study was found to decrease with increase in age. Pregnant women who were between the ages of 15 -24 were more malnourished than those in the age categories of 35-44 years. This is contrary to studies in Tanzania that showed that women within the age brackets 20-29 years and 30-39 years were less likely to suffer from under nutrition compared to women in the age brackets 15-19 years and 40-49 years [18]. It is difficult to explain the reason for this observation. This study also shows that women who were in the age category 25-34 years were more anemic compared to those who were in the age category $35-44$ years. This is comparable to a study conducted in Osogbo by Wasiu and Olufemi [19] those with non-education and tertiary education. However, result of a similar study conducted in Tanzania [18] showed that those with primary education were less likely to be affected by chronic under nutrition than women with secondary or higher education. According to NDHS 2013 data on nutritional status of women, women with low education were more malnourished than those with primary or higher education [14]. Also the result of DHS conducted in Ethiopia in 2000 showed that malnutrition among pregnant women decreased with increase in level of education. Another study conducted by Olujimi et al [20] showed that the proportion of anaemic women increased with decrease in the level of education.

The findings in this study revealed that among pregnant women in Odo-Otin Local government Area, civil servants were more malnourished than farmers. This is unlike the result of findings in the study conducted by Olujimi et al [20] which showed that women of high socio-economic status had low prevalence of malnutrition. The study shows that those who earn less than N10, 000.00 were more malnourished than those who earn more than N50,000.00. This is in accordance with studies that showed that women of high social class had good nutri- tional status than those who were less economically empowered [20].

This study has revealed that the prevalence of malnutrition among pregnant women in Odo-Otin Local Government area was low. The study also revealed that there were significant associations between the sociodemographic factors, economic status and nutritional status. The result of the analysis confirmed that there was association between the dietary practice and nutritional status of the pregnant women. This is obvious as majority of the participants who skipped meal were malnourished, under-weight, had MUAC less than $23 \mathrm{~cm}$ and were anemic. Also, pregnant women who were civil servants in Odo-Otin local government area, Osun state were more malnourished and anemic than farmers and artisans. It is therefore recommended that the state government should consider improvement in the salaries of civil servants and restore their full salaries. Government should also sponsor special dietary education sessions and, make available subsidized balanced energy and protein supplements especially for the pregnant women.

\section{Acknowledgement}

Our appreciation goes to Dr. S. O. Sobaloju, Oba elect of Alapata in Ile-Ife, Dr. Adeniyi Oginni of Osun State Ministry of Health, and Dr. Ogundiran Abdulrahman for their advice, encouragement and help in reviewing some parts of the study. Our appreciation also goes to PHC coordinator in Odo-Otin Local Government Area, Dr. Olaogun, all Nurses and Community Health extension workers at all the Primary Health Care Centers where the data were collected. We also appreciate the effort of matron and staff of Primary Health Care Center, Dada Estate, Osogbo, Osun State where the questionnaires were pre-tested.

\section{Conflict of interest}

The authors declare that there is no conflict of interest

\section{Authors' Contributions}

AA performed data collection. All authors conceptualized the study, performed data analysis, drafted, revised and approved the final manuscript.

\section{References}

1. Diet and nutrition: Healthy eating and balanced diets tips. Available at www.emedicinehealth.com/ nutition_and_diet/article_em.htm. Accessed 4th October, 2016.

2. Ojofeitimi EO. Some key definition of nutrition terminologies with explanations. In principles and practices of nutrition for public health practitioners. $5^{\text {th }}$ eds. Ibadan: None- 
such house publishers; 2014; p.27-35.

3. Franca Marangoni, Irene Cetin, Elvira Verduci, Giuseppe Canzone, Marcello Giovannini, Paolo Scollo Franca Marangoni1NFI-Nutrition Foundation of Italy, Milano 20124, Italy; ti.noitadnuof-noitirtun@ilopet al. Maternal Diet and Nutrient Requirements in Pregnancy and Breastfeeding. Nutrients. 2016; 8(10): 629.

4. Find articles Household food security status 'In' Dietary Diversity and Nutritional Status of Pregnant Women Aged 15-45 years Attending Kapengura District Hospital West Pokot Country Kenya. Available at ir-library.ku.ac.ke/ bitstream /handle/.../7486/marital\%2ol Lillian\% kemuntopdf?.accessed 4th October, 2016.

5. Ogbodo SO, Nwagha UI, Okaka An, Ogeniyi SL, Okoro RO, Chukwurah FE. Low Levels of Some Nutritional Parameters of Pregnant Women in a Rural Community of South East Nigeria: Implication for The Attainment of the Millennium Development Goals. Ann Med Health Sc. Res. 2012; 2 (1): 49-55

6. Oluwole OB, Agboola AA, Adeyoju OA, Onyibe and Kayode F. Improving maternal malnutrition in Nigeria. EC nutrition. 2016; 1.SI S4-S9.

7. Janice LT, Lind AU, Mehada MM. Nutrition Through The Life Cycle: Pregnancy and The First year of Life 'In' The Science of Nutrition. 2nd eds.. USA: Pearson Benjamin Cummings Publishers; 2011; p. 623.

8. Srialakshm B. Nutritional and food requirement of expectant mother 'In' dietetics.7eds. New Delhi: New age international (p) Limited Publisher; 2014; p.166-134.

9. Haider, Batool AB, Zulfiqar A. "Multiple-micronutrient supplementation for women during pregnancy". The Cochrane Database of Systematic Reviews. 2017; 4: CDo04905. doi:10.1002/14651858. CDoo4905. pub5. ISSN 1469-493X. PMID 28407219.

10. Ota E, Hori H, Mori R, Tobe-Gai R, Farrar D,. "Antenatal dietary education and supplementation to increase energy and protein intake". The Cochrane Database of Systematic Reviews. 2015; 6:

CDoooo32.
doi:10.1002/14651858.CDoooo32.pub3. PMID 26031211.

11. History of Odo-Otin Local Government Area. Available from wikipedia.org/

12. History of Osun State. Available from Wikipedia.org/wiki/ osun-state, Accessed 4th October, 2016.

13. Population Commission (NPC) [Nigeria] and ICF International. Nigeria Demographic and Health Survey National 2013. Abuja, Nigeria, and Rockville, Maryland, USA: NPC and ICF International; 2014; p.195-197.

14. National Bureau of Statistics [NBS] and National Population Commission [NPC]. Available at www.nbuneolineg.com/nbs/2016. Accessed December 2016.

15. Israel GD. Determining sample size. University of Florida. IFAS Extension 1992; PEOD6.

16. Sarah JP, Ana M, Ortega-Villa, William G, Stefanie NH, Roger BN, Mary $\mathrm{H}$ et al. Longitudinal changes in maternal anthropometry in relation to neonatal anthropometry. Public Health Nutr. 2019 Apr; 22(5): 797.

17. Ikechukwu OO, NgwuNwachukwu, Emmanuel IU, Obas UO, Protein Energy malnutrition among urban and rural pregnant women in Ebonyi State, South Eastern Nigeria. International Journal of Health Research. 2012; 5 (2): 5360.

18. Mutumwa AH, Paul E, VuaiSah. Determinant of Under nutrition among women of reproductive age in Tanzania Mainland. South African Journal of Clinical Nutrition. 2016; 29 (2): 75-81

19. Wasiu OA and Olufemi EA. How valid is the Tallquist Method in the Screening of Pregnant women with Anaemia in Poor Rural Setting of South Western Nigeria? Med J. Islam Repub. Iran. 2016; 30: 389.

20. Olujinmi AO, Aniekan MA, Emem AB, Robert SJ, Godwin I, Anyiekere M, . Prevalence of Anemia Among pregnant Women at Booking in University of Uyo Teaching Hospital, Uyo, Nigeria. Bio-Med Research International. 2014; 8 pages. 